\title{
Group B Streptococcal Meningitis: A Description of Six Case Reports
}

\author{
Elisabetta Coni ${ }^{\mathrm{a}}$, Maria Antonietta Marcialis ${ }^{\mathrm{a}}$, Maria Cristina Pintus ${ }^{\mathrm{a}}$, Roberta Irmesia ${ }^{\mathrm{a}}$, Valentina Masile ${ }^{\mathrm{a}}$, \\ Vassilios Fanos ${ }^{\mathrm{a}, \mathrm{b}}$
}

\begin{abstract}
Group B Streptococcus (GBS) is considered the leading bacterial cause of neonatal infections and meningitis is the most serious manifestation. Its clinical manifestations are usually non-specific and its signs and symptoms may be subtle, unspecific, vague, atypical or absent. We report six clinical cases of GBS meningitis with early onset in three infants (first week of life) and late onset (after 1 month of life) in the remaining three. Prenatal GBS screening, performed on four infants, resulted positive only in one case. Cerebral ultrasonography showed high sensitivity in detecting early meningeal involvement confirmed by MRI in all infants. Our study describes the clinical characteristics, antibiotic sensitivity and outcome of the six cases. The results of our analysis emphasize the severity of GBS meningitis and show that cerebrovascular involvement is a common but poorly studied pathologic process in GBS meningitis. In addition, cranial ultrasonography plays an important role in detecting infarctions, cerebritis, hemorrhages or abscesses and in monitoring complications. Neonates with GBS meningitis are prone to adverse outcomes or sequelae. Mortality in GBS meningitis approaches 30\% and neurologic sequelae are present in $25 \%$ of cases.
\end{abstract}

Keywords: Group B Streptococcus; Neonatal meningitis; Cerebral ultrasonography

\section{Introduction}

Group B Streptococcus (GBS) is the leading bacterial cause of serious infections in the neonatal period, along with $E$. coli and L. monocytogenes; GBS is responsible for $70-80 \%$ of bacterial

Manuscript accepted for publication January 21, 2015

aDepartment of Surgery, Neonatal Intensive Care Unit, Puericulture Institute and Neonatal Section, Policlinico Monserrato, Azienda Ospedaliera Universitaria di Cagliari, University of Cagliari, 09042 Monserrato, Italy

${ }^{\mathrm{b}}$ Corresponding Author: Vassilios Fanos, NICU, Puericulture Institute and Neonatal Section, Polic linico Univers itario dell' Azienda Ospedaliera Universitaria di Cagliari, Blocco Q, Strada Statale 554, Bivio Sestu, 09042 Monserrato (Cagliari), Italy. Email: vafanos@tiscali.it

doi: http://dx.doi.org/10.14740/ijcp179w meningitis [1]. GBS is a Gram-positive encapsulated commensal bacterium of the human intestine, which is also present in $15-30 \%$ of healthy women's vaginas. Fifty percent of infants born from colonized mothers are asymptomatic at birth, but only $1 \%$ of them develop invasive disease (sepsis, meningitis, pneumonia, osteomyelitis, septic arthritis, cellulitis, and otitis) [2]. GBS infections can be classified as an early-onset disease (EOD), occurring within the first week of life, and late-onset disease (LOD), between the second week and 3 months of age.

\section{Clinical features of GBS meningitis}

GBS meningitis is considered the most serious manifestation of LOD infection, although sometimes it can occur earlier, just a few hours after birth. Together with Gram-negative infections, GBS has the worst prognosis.

The early symptoms may be unspecific and include temperature instability, feeding difficulties, excessive crying or irritability, respiratory distress. Signs and symptoms of meningitis may be subtle, unspecific, vague, atypical or absent. The early signs and symptoms of neonatal meningitis are similar to those of septicemia: lethargy, feeding problems, altered temperature, respiratory distress, apnea, jaundice and abdominal distention. Further on, stiff neck, hypo/hypertonia, bulging fontanel and seizure may appear.

\section{Pathogenesis}

GBS is classified into eight serotypes (Ia, II, III, IV, V, VI, VII, and VIII) on the basis of capsular polysaccharide and presence/ absence of $\mathrm{c}$ and $\mathrm{R}$ protein antigens. The EOD can be caused by all serotypes while serotype III is the one most responsible for LOD (90\% of cases) [3-5]. Furthermore, serotype III is the one mainly responsible for cases of GBS meningitis, regardless of the age at onset of infection [6]. The development of GBS meningitis reflects the ability of the bacteria to invade the microvascular endothelial cells of the human brain (brain microvascular endothelial cells (BMECs)). The first step in the pathogenesis of meningitis consists of the interaction between GBS and BMECs. GBS can gain access through the CNS by direct lysis of BMECs with consequent endothelial damage, inflammation and disruption of the integrity of the BBB or, alter- 
natively, by bacterial transcytosis. Several virulence factors are thought to be involved in the pathogenesis of meningitis: SRR1 and SRR2 (serine-rich repeat glycoprotein) [7, 8], the HvgA (hypervirulent GBS adhesin) [9], the Lmb (laminin-binding protein), and LagA (lipoteichoic acid anchoring enzyme) [10].

Reactive nitrogen species are the most important mediators of brain inflammation [11]. Eventually, GBSs can reach the brain through choroid plexus or intraventricular hemorrhage [11]

\section{Diagnosis}

The analysis of cerebrospinal flow (CSF) is the gold standard for the diagnosis of GBS meningitis.

Cranial ultrasonography (US) plays an important role in the initial evaluation of an infant with suspected bacterial meningitis as well as in monitoring all possible complications. Yikilmaz et al [12] recommend cranial US as a first study in infants who have an adequate size of fontanel if there is a clinical suspect of bacterial meningitis. MRI should be the next study of choice [12].

\section{Prognosis and outcome}

Mortality of GBS meningitis approaches 30\% [13] and longterm evaluation of infants who survive GBS meningitis indicates that $30 \%$ of cases have mild-to-moderate neurologic sequelae and 19\% have severe sequelae with global cognitive delay, cerebral palsy, cortical blindness, and/or hearing impairment [14].

\section{Cranial US}

Initial sonographic anomalies (hours or few days)

1) Brain swelling with slitlike lateral ventricles and swollen and hyperechoic choroid plexus due to plexitis.

2) Hyperechoic sulci and increased echogenicity of the cortex related to exudation.

\section{Possible evolution in an intermediate stage (second week)}

1) Dense ventricular lining due to ependymitis.

2) Inhomogeneous echogenicity in the white matter due both to inflammation and infarction.

3) Thalami and basal ganglia nodular focal infarcts due to thrombophlebitis.

4) Initial subtle intraventricular strand formation (ventriculitis).

\section{Advanced stage}

Periventricular, intermediate and subcortical cysts due to com-

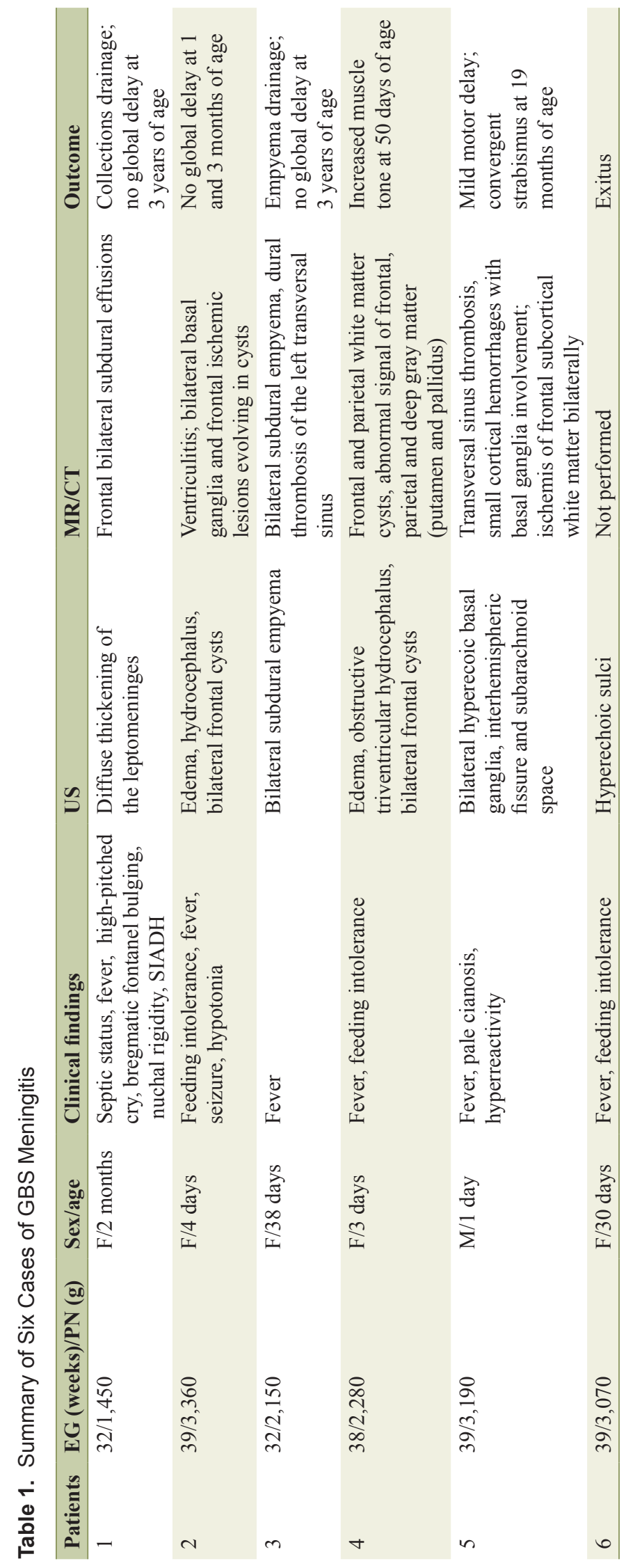




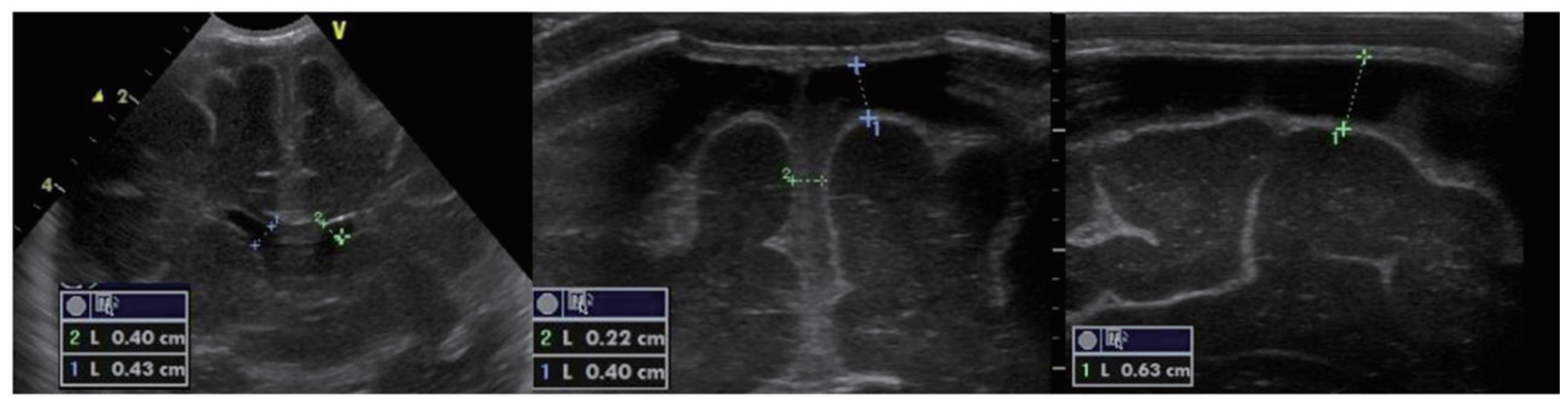

Figure 1. Echogenic widening of brain's sulci and interhemispheric fissure associated with meningeal thickening; mild ventricular enlargement.

bination of infection and venous infarction.

\section{Complications}

1) Widening of the ventricles due to obstruction of CSF evolving into hydrocephalus.

2) Subdural effusion, either sterile or purulent, which appears as round biconvex collection.

\section{Case Reports}

We describe six case reports of GBS meningitis: three cases of
EOD and three cases of LOD. The population is summarized in Table 1. Case details are provided below. Prenatal GBS screening through vagino-rectal swab was performed in four cases: it resulted negative in three cases and positive in one case. In two cases (one preterm and one term newborn), the screening was not performed.

Median age at onset of symptoms was 23.1 days (range 1 - 63 days).

\section{Case 1}

A preterm female newborn (32 weeks, 1,485 g), second of triplets, was born from a healthy mother. GBS screening was

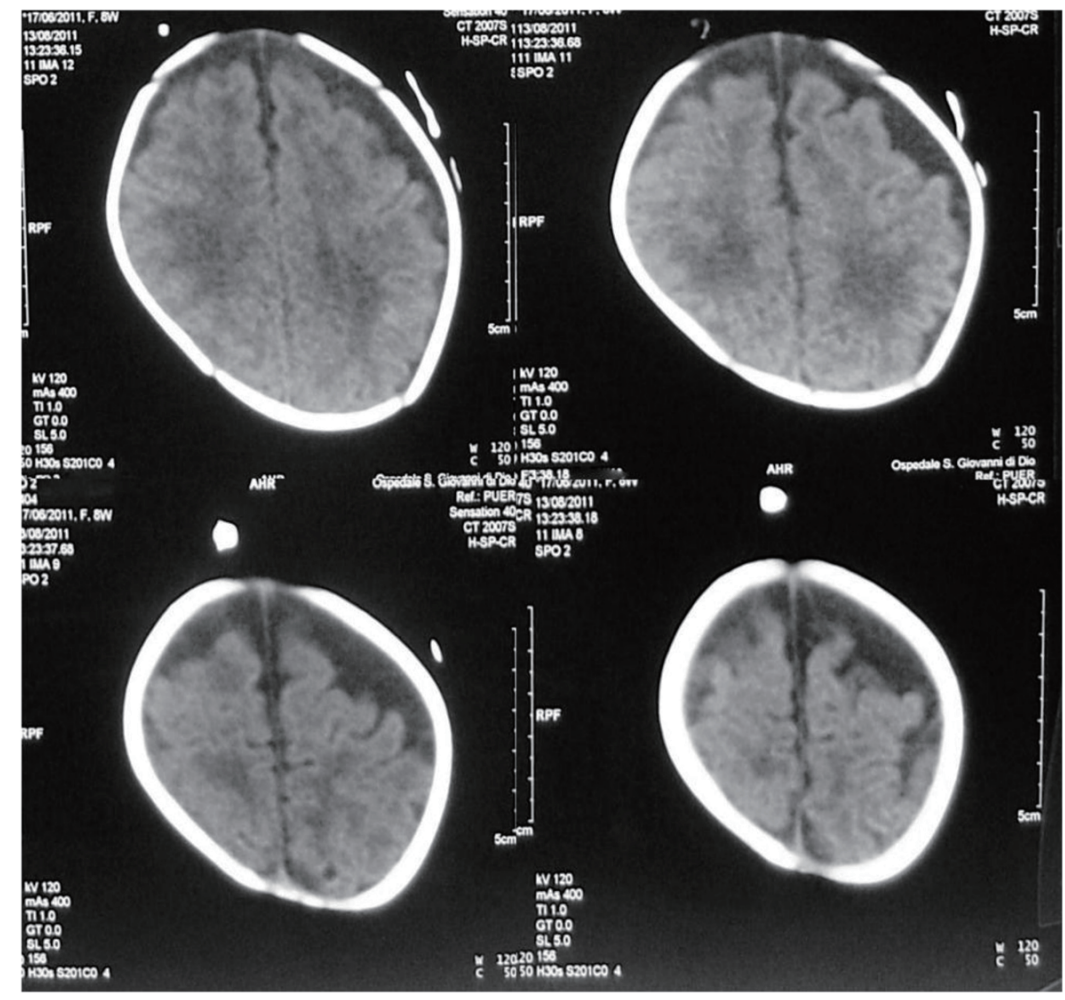

Figure 2. Brain MRI: bilateral asymmetrical subdural effusions, prevalent on the right side of the brain. 


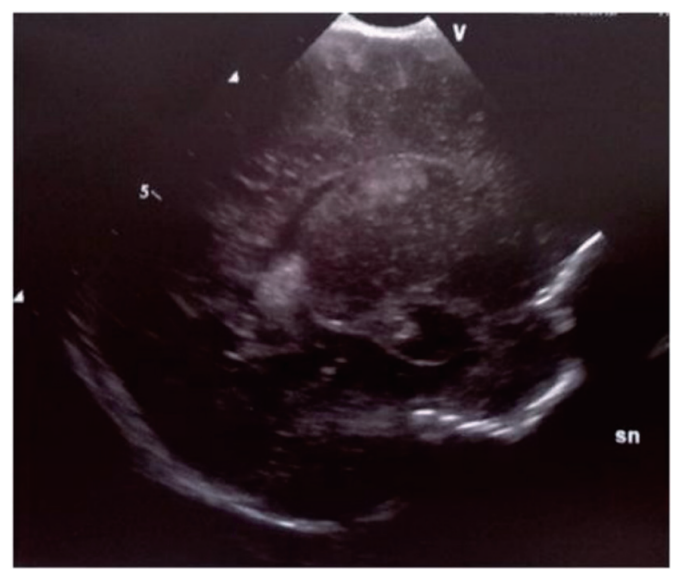

Figure 3. Parasagittal section: multiple echodensities of the left caudate.

not performed. She was admitted to neonatal intensive care unit (NICU) and discharged 1 month later in a good general state. At about 2 months of age, she was readmitted for fever, septic, bulging bregmatic fontanel, stiff neck and high-pitched cry, followed by seizure. The initial blood test revealed leukopenia (WBC 2,400/mmc; N 1.27\%), increased C-reaction protein (CRP, > $200 \mathrm{mg} / \mathrm{L}$ ) and procalcitonin (PCT, > $100 \mathrm{ng} /$ $\mathrm{mL}$ ), positive blood and CSF culture for GBS. CSF revealed pleocytosis $(160$ cell $/ \mu \mathrm{L})$ and increased protein $(111 \mathrm{mg} / \mathrm{dL})$. Treatment with ampicillin, gentamicin and ceftazidime was started. The course of disease was complicated by SIADH (Na $121 \mathrm{mEq} / \mathrm{L})$. To treat seizure, sodium supplementation and anticonvulsant therapy were required. Cranial US revealed echogenic widening of brain sulci and interhemispheric fissure associated with meningeal thickening together with mild ventricular enlargement (Fig. 1). Brain MRI revealed frontal bilateral subdural effusions (Fig. 2).

The infant was transferred to a neurosurgical unit to drain the collections. Neurological evaluation at 4 years of age demonstrated no global delay and no visual or auditory impair-

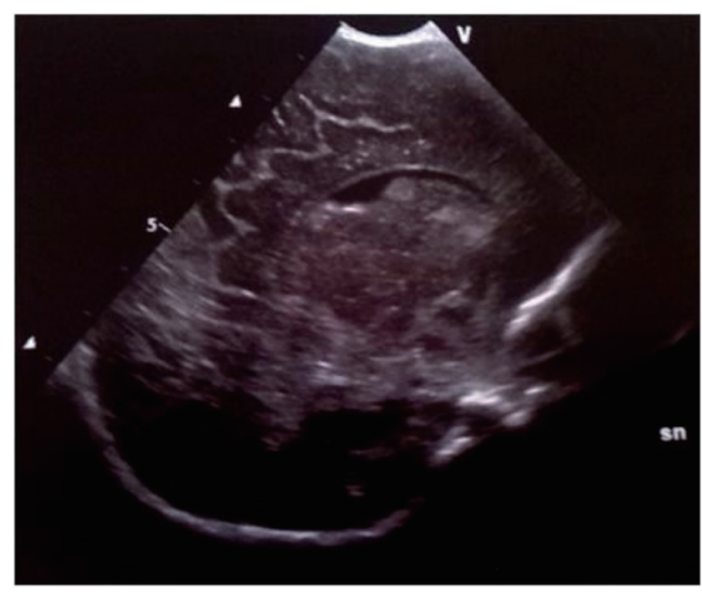

Figure 4. Parasagittal section: multiple echodensities of the caudate head.



Figure 5. Coronal section: bilateral echodensities of the caudate and of the right periventricular white matter.

ment.

\section{Case 2}

A term female newborn (39 weeks, PN 3,360 g) was born by vaginal delivery after physiologic gestation. GBS screening was performed at 35th and 37th week and resulted negative. At 4 days of life, she was admitted to the NICU for fever, feeding intolerance, poor perfusion, hypotonia, whiny crying and seizure. Immediate treatment with double anticonvulsant therapy was started. The initial blood analysis revealed increased CRP (107 mg/L) and PCT (9.85 ng/mL) and positive blood culture. Nasal and pharyngeal swabs and CSF culture were positive for GBS. Triple antibiotic (ampicillin, gentamicin and ceftazidime) treatment was started. The first cranial US (Fig. 3 ) was performed at 4 days of life. US revealed a markedly hyperechoic right thalamus and basal ganglia. After 2 days a second scan showed another echodensity within the caudate head (Fig. 3). Subsequently, multiple foci of cortical and subcortical hyperechogenicities were observed (Fig. 4). The last scan, performed at 2 months of life, revealed an irregular and asymmetrical ventricular enlargement with a notch of ependyma just above the left thalamus (Fig. 5). MRI (Fig. 6) demonstrated ventriculitis and bilateral basal ganglia and frontal ischemic lesions which evolved into multiple cysts in 20 days. Neurological evaluation at 1 and 4 months demonstrated normal findings.

\section{Case 3}

A preterm female (32 weeks, 2,150 g) was born after gestation complicated by gestational diabetes. SGB screening was negative. She was admitted to the NICU and discharged 1 month later in a good general state. Four days later, because of the onset of fever, she was brought in for care. The initial blood analysis revealed leukocytosis, increased CRP (100 mg/L) and PCT $(36 \mathrm{ng} / \mathrm{mL})$ and positive blood culture for GBS. CSF analysis demonstrated pleocytosis $(400 \mathrm{cell} / \mu \mathrm{L})$, hypoglycor- 


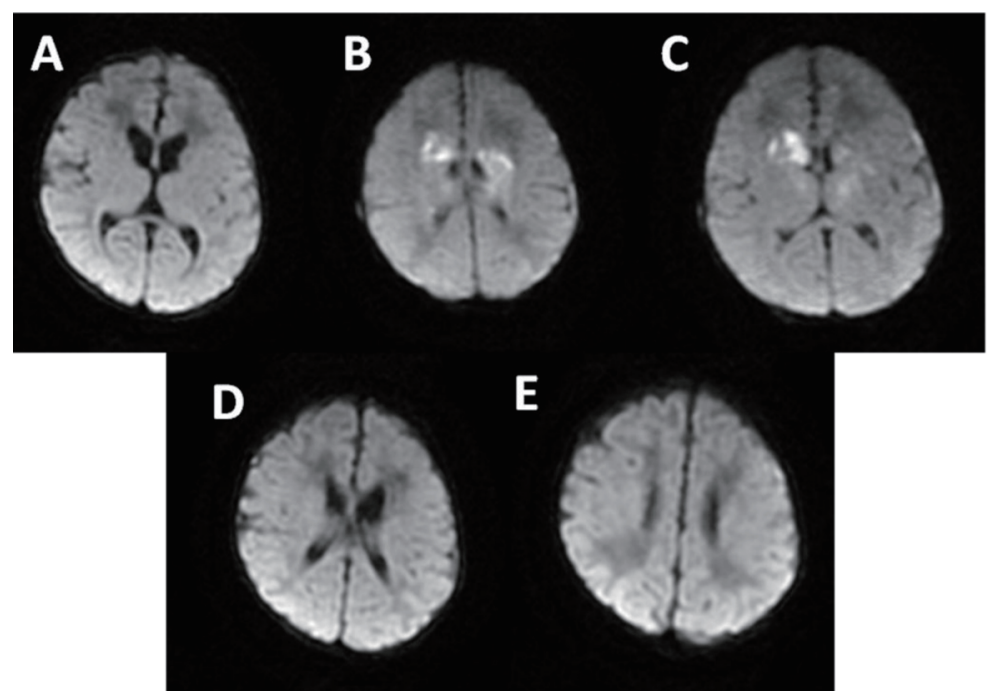

Figure 6. MRI: multiple and confluent ischemic areas $(B-C)$ in the basal ganglia and frontal periventricular white matter.

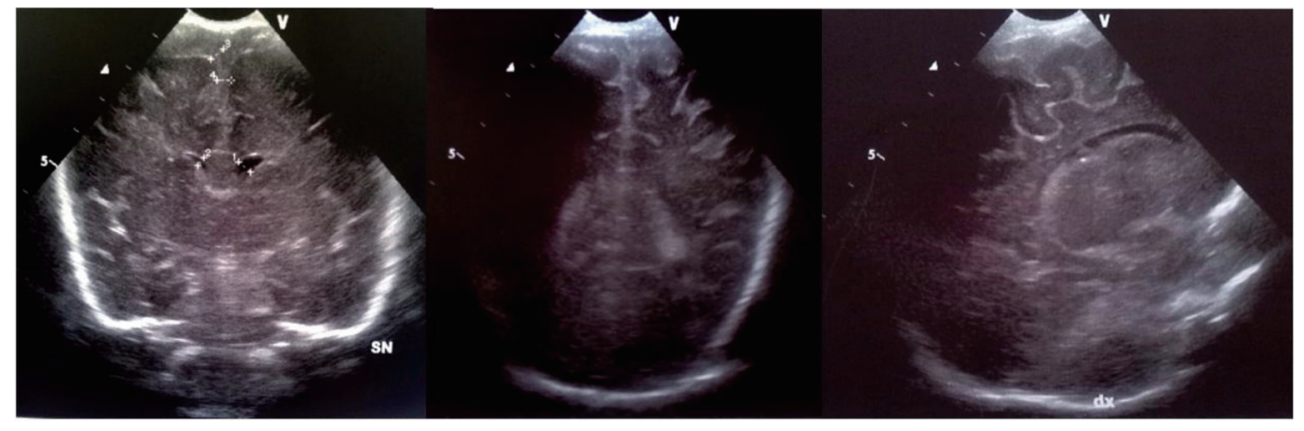

Figure 7. Echogenic widening of brain sulci and interemispheric fissure.

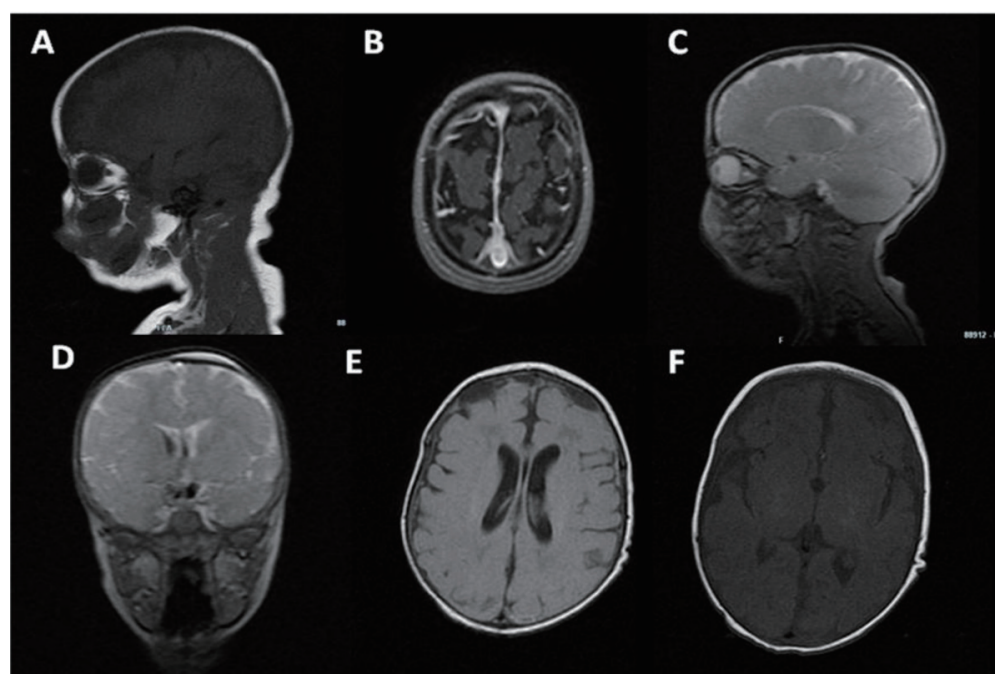

Figure 8. MRI: biconvex subdural effusions (A-E); strand formation within the lateral ventricles (D-E); ischemic foci in the basal ganglia $(C, F)$; bilateral frontal periventricular ischemic areas $(E)$. 


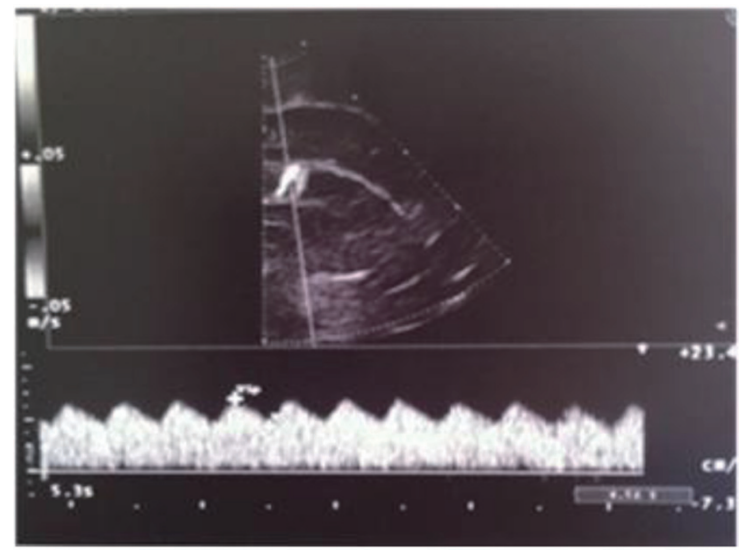

Figure 9. Abnormal resistance index $(\mathrm{RI}=0.35)$.

rhachia (21 mg/dL) and increased protein (346 mg/dL); Grampositive cocci were present. Double antibiotic treatment (gentamicin and ceftazidime) was started.

Cranial US (Fig. 7) revealed widening of the subarachnoid space with hyperechoic debris content. MRI demonstrated widening supratentorial liquoral spaces, subdural collections and left transversal sinus thrombosis (Fig. 8).

The infant was transferred to a neurosurgical unit to drain the empyema. The neurological evaluation at 3 years of age demonstrated no global delay and no visual or auditory impairment.

\section{Case 4}

A term female newborn ( 38 weeks, 2,280 g) was born by cesarean section $12 \mathrm{~h}$ after membrane rupture. GBS screening was not performed. The mother had an uneventful pregnancy. At 3 days of life, the newborn manifested fever, poor general conditions, poor perfusion, whiny crying and feeding intolerance. She was admitted to the NICU. The initial blood analysis revealed leukocytosis (WBC 27,700/mmc; N 87\%), increased CRP (270 mg/L), PCT (104 ng/mL) and presepsine (3,432 pg/

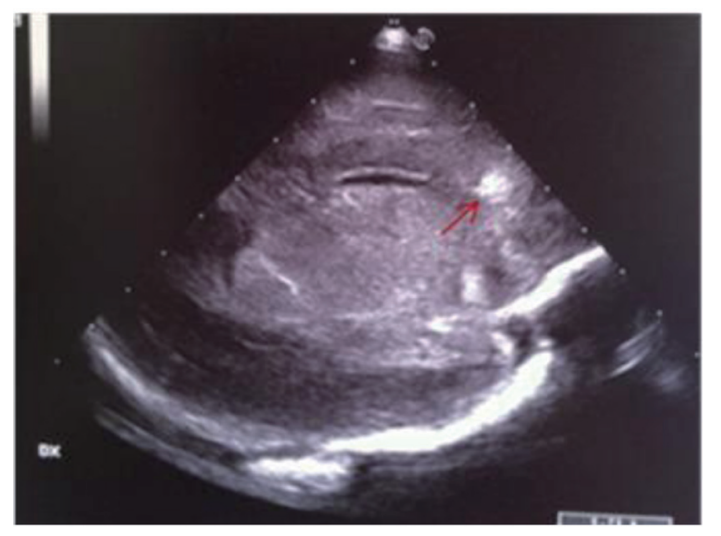

Figure 10. Parasagittal section: frontal periventricular echodensity (red arrow).

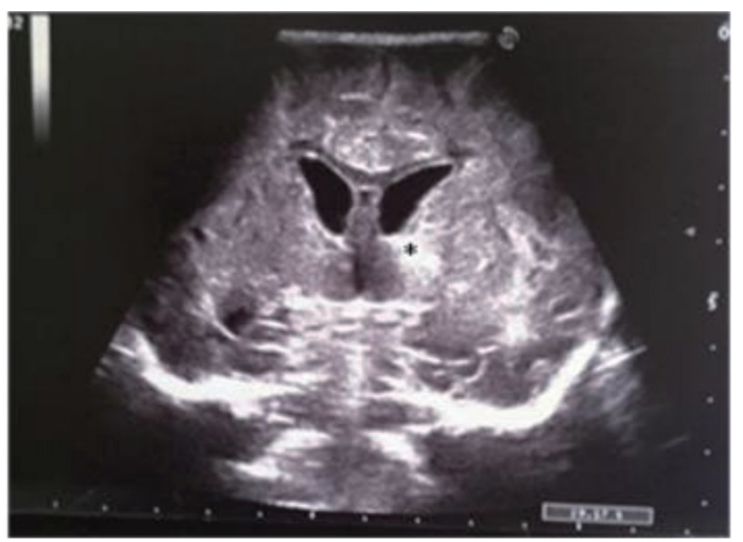

Figure 11. Coronal section: asymmetric widening of lateral ventricles; single and localized echodensity in the left thalamus (black star).

$\mathrm{mL}$ ). Blood culture, nasal and pharyngeal swabs were positive for GBS. Initially, the CSF analysis was not performed. Double antibiotic treatment was started. Cranial US revealed a very low resistance index $(\mathrm{RI}=0.35)$ and right frontal periventricular echodensity (Fig. 9, 10). Twenty-four hours later, she developed generalized hypertonia and clonic seizure that resolved with double-drug anticonvulsant therapy. The CSF analysis revealed hypoglychorrachia $(15 \mathrm{mg} / \mathrm{dL})$, pleocytosis (1,920 cell/mmc with predominant neutrophils), and increased protein $(403 \mathrm{mg} / \mathrm{dL})$; CSF culture was positive for GBS. Because of the clinical deterioration, cranial US was repeated; it revealed asymmetric widening of lateral ventricles [12-15], single and localized echodensity in the left thalamus (Fig. 1113) and a single intraventricular strand (Fig. 12, 14), followed in the last scan by obstructive triventricular hydrocephalus and bilateral frontal cysts (Fig. 15-18). MRI (Fig. 19) demonstrated the presence of frontal and parietal white matter cysts, abnormal signal of frontal, parietal and deep gray matter (putamen and pallidus). Because of the evolving hydrocephalus, the infant was transferred to the neurosurgical unit. The clinical evaluation at 50 days of life showed increased muscle tone.

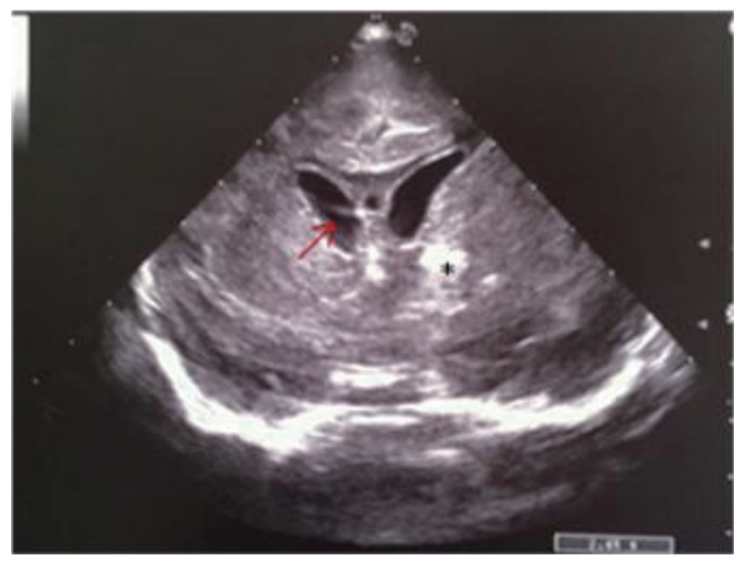

Figure 12. Coronal section: asymmetric widening of lateral ventricles; intraventricular strand (red arrow); localized echodensity in the left thalamus (black star). 


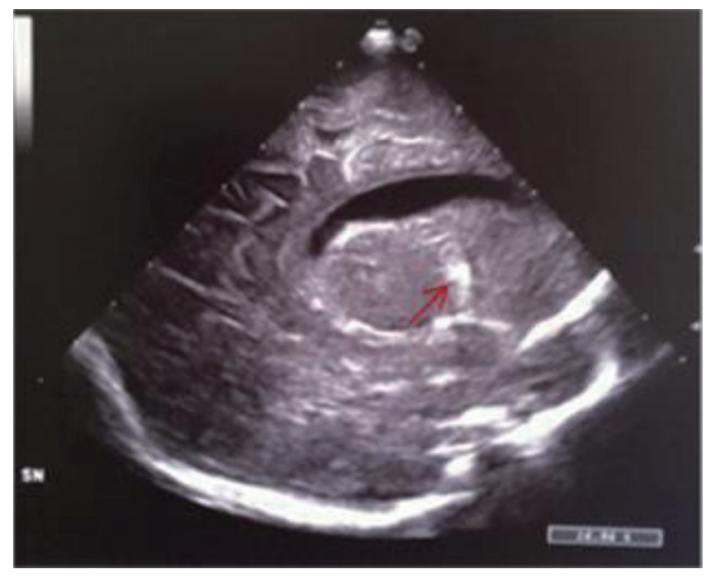

Figure 13. Median parasagittal section: widening of the left lateral ventricle and localized echodensity in the left thalamus (red arrow).

\section{Case 5}

A male term newborn (39 weeks, 3,190 g) was born by vaginal delivery after an uneventful pregnancy. GBS screening was negative at 37 weeks' gestation. A few hours later, because of the development of pale cyanosis followed by fever and hyperreactivity, he was admitted to the NICU. The initial blood analysis revealed leukopenia (WBC 3,100/mmc; 75\% neutrophils), increased CRP $(168.9 \mathrm{mg} / \mathrm{L})$ and PCT $(21.5$ $\mathrm{ng} / \mathrm{mL}$ ). CSF demonstrated pleocytosis (420 cell $/ \mathrm{mmc}$ ), hypoglycorrhachia $(3 \mathrm{mg} / \mathrm{dL})$, and increased protein $(420 \mathrm{mg} /$ $\mathrm{dL}$ ); CSF and the blood culture were positive for GBS. Triple-antibiotic therapy was started. The first cranial US was completely normal (Fig. 20); 3 days later, the scan showed a mild widening of interhemispheric fissure with subarachnoid echogenic strands (Fig. 21), particularly severe near the occipital regions (Fig. 22), asymmetric lateral ventricles, rounded echodensity in the left thalamus and incipient cavitation of both left caudate nucleus and thalamus (Fig. 23). MRI revealed small hemorrhages in the thalami and right transversal sinus thrombosis together with ischemic-hemorrhagic foci in

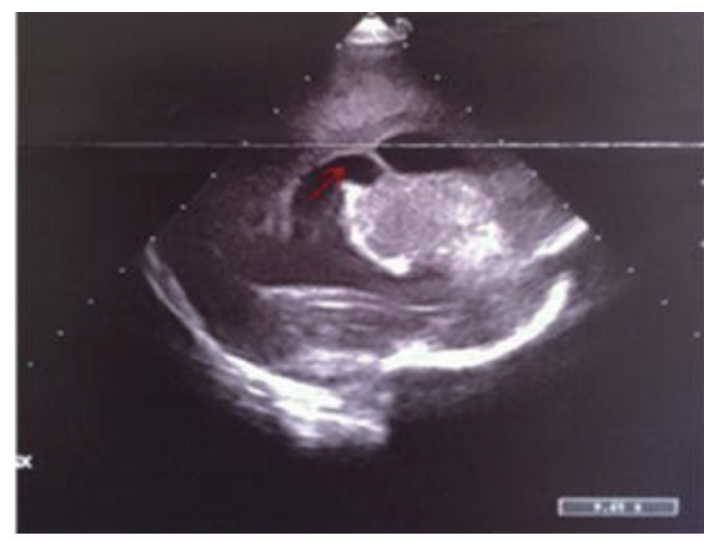

Figure 14. Left parasagittal image: widening of lateral ventricle with a septum inside (red arrow) and localized echodensities of anterior thalamus.

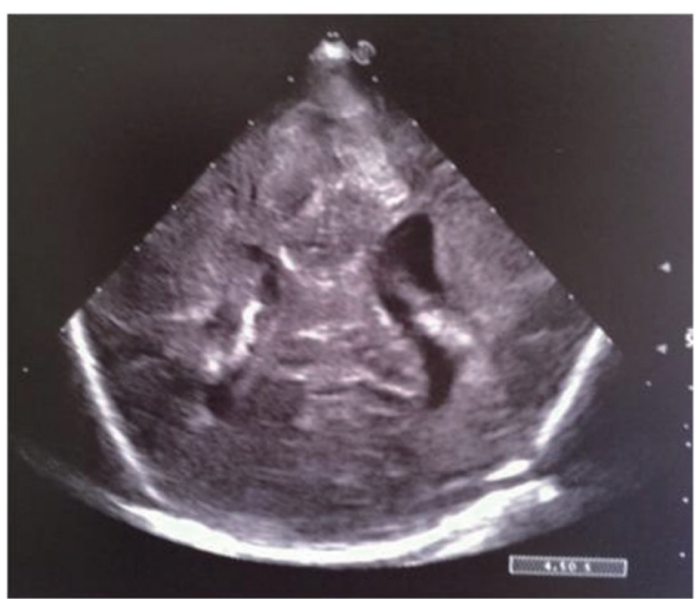

Figure 15. Coronal section: asymmetric widening of lateral ventricles.

the frontal subcortical white matter bilaterally (no available image).

\section{Case 6}

The last girl was born at term ( 39 weeks, $3,070 \mathrm{~g}$ ) by vaginal delivery after a physiologic pregnancy. GBS screening was positive; a single dose of antibiotic (ampicillin) was given to the mother a few hours before giving birth. At 25 days of life, because of the development of fever and feeding intolerance, the newborn was brought in for care. She presented poor perfusion, decreased level of consciousness, hypotonia and seizure. The initial blood analysis revealed leukopenia (WBC 2,500/ $\mathrm{mmc} ; 45 \%$ neutrophil and 51\% lymphocytes), hypoglycemia $(36 \mathrm{mg} / \mathrm{dL})$, increase PCT $(338.5 \mathrm{ng} / \mathrm{mL})$ and presepsine $(762$ $\mathrm{pg} / \mathrm{mL})$. CSF revealed hypoglycorrhachia $(3 \mathrm{mg} / \mathrm{dL})$, absence of cells, increased protein concentration $(283 \mathrm{mg} / \mathrm{dL})$. CSF and blood culture were positive for GBS. Double antibiotic (gentamicin, ampicillin + sulbactam) therapy was started. Cranial sonography demonstrated hyperechoic sulci between the gyri (no available images). Progressively, she worsened and

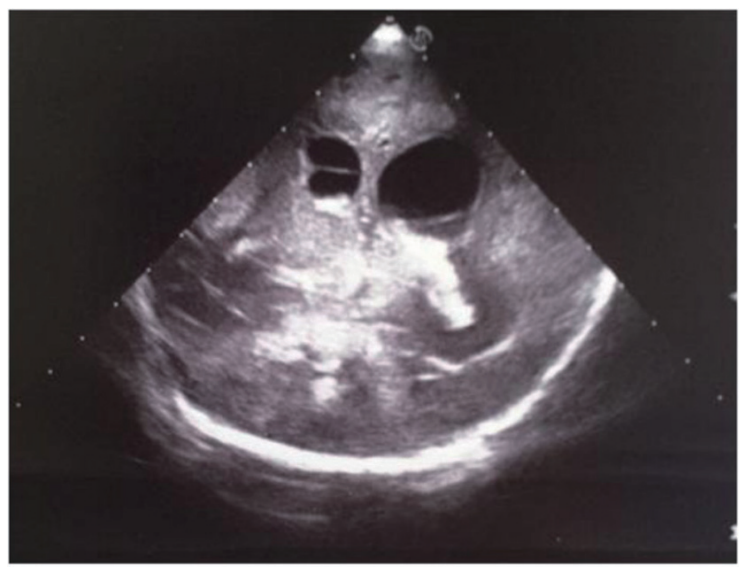

Figure 16. Coronal section: asymmetric widening of lateral ventricles; intraventricular strand; localized echodensity in the left thalamus. 


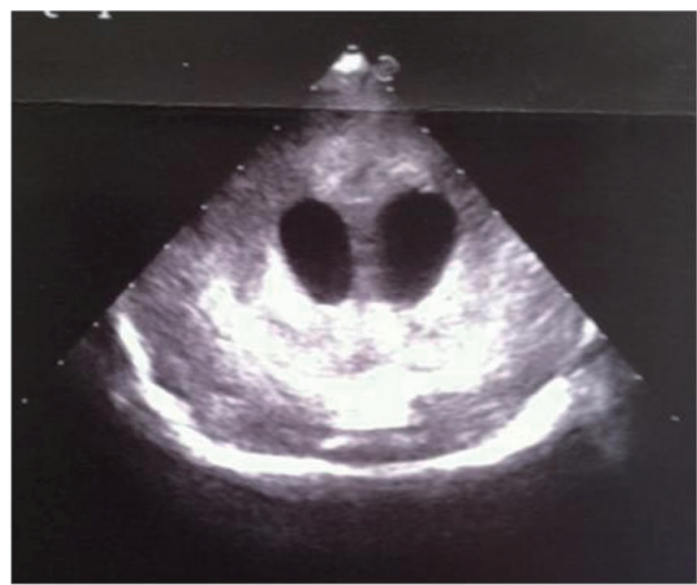

Figure 17. Coronal section: widening of the lateral ventricles and hyperechoic basal ganglia.

died $12 \mathrm{~h}$ after admission.

\section{Discussion}

GBS is the main bacterial cause of serious infection in newborns. The guidelines for the prevention of perinatal GBS disease provided by the CDC (Centers for Disease Control and Prevention) in 2002, recommend performing rectal and vaginal swabs between the 35th and 37th week of gestation and intrapartum antibiotic prophylaxis (IAP) in colonized women [15]. According to the American Academy of Pediatrics [4], IAP has managed to reduce by $80 \%$ the incidence of GBS EODs, but has not shown significant efficacy in the prevention of LODs [9]. The vagino-rectal swab identifies the presence of GBS in $54-87 \%$ of cases and it is therefore possible to have some false negatives [16]: in our study GBS screening was negative in three cases. The maternal status for GBS cannot be used as the only risk factor for GBS EOD



Figure 18. Sagittal section: severe widening of the left lateral ventricle and hyperechoic basal ganglia.

and LOD. Furthermore, it is important to emphasize that the guidelines recommend vagino-rectal swab performance between the 35th and 37th weeks. As a consequence, in the case of a premature birth, maternal GBS status may be unknown [17]. Horizontal transmission and environmental sources (nosocomial and familiar) are the main causes of GBS LOD; thus, the association of vagino-rectal swab and LOD is controversial.

GBS meningitis is considered the most serious manifestation of LOD infection, but sometimes it can occur in the first week of life: in our study, meningitis was a manifestation of LOD in three newborns but in the other three cases, meningeal involvement occurred in the first week of life; one of them developed this condition just a few hours after birth.

Cranial US is an important tool for the initial evaluation of an infant with suspected neonatal meningitis and for monitoring its evolution. Although in the earliest stages the findings may be normal (as in our case 5), US may detect the presence of edema, ventriculitis, liquoral space widening, subdural effusion, focal or diffuse parenchymal involvement by cerebritis,

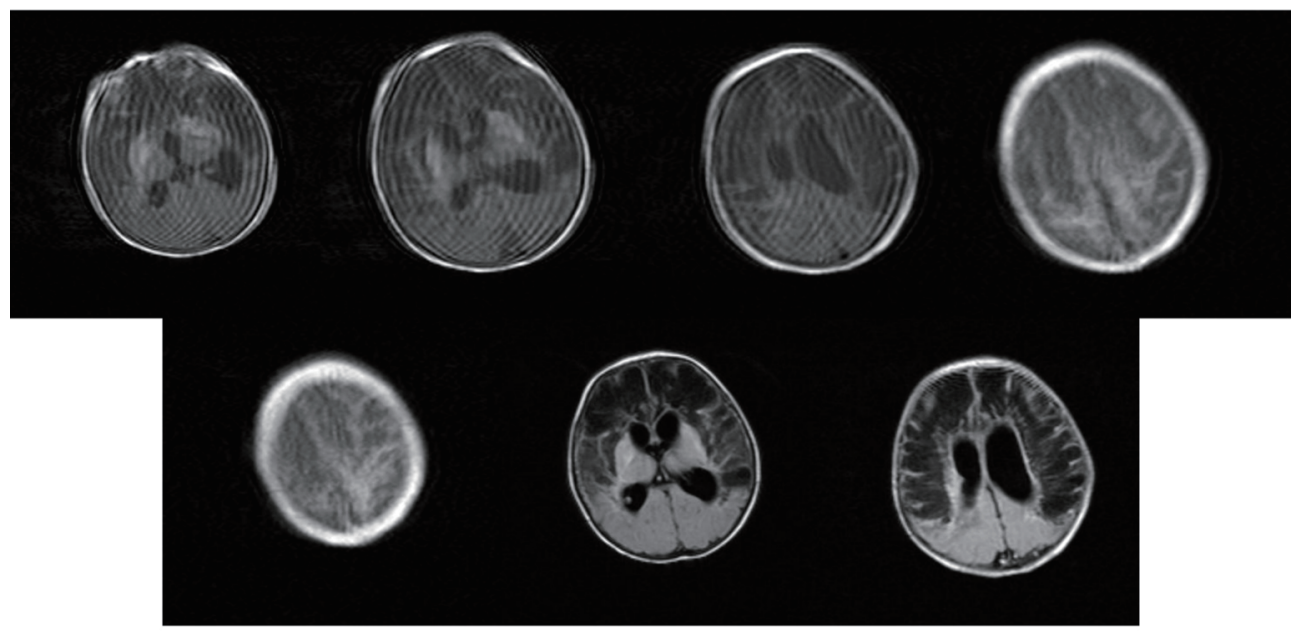

Figure 19. MRI: Movement artfacts. Bilateral multiple extensive cysts in the fronto-parietal white matter and focal bilateral emorrhages of lenticular nucleus. Obstructive triventricular hydrocephalus. 




Figure 20. Normal resistance index (IR) but evidence of increased telediastolic velocity.



Figure 21. Coronal section: mild widening of interhemispheric fissure with subarachnoid echogenic strands.

infarction, hemorrhages or abscesses.

Contemporary long-term outcomes for children surviving GBS meningitis reveal that $56 \%$ are functioning normally. The remainder undergoes mild-to-moderate $(25 \%)$ or severe (19\%) neurodevelopmental impairment [18] such as cerebral palsy, language and cognitive impairments, deafness, vision disorders, epilepsy and hydrocephalus. Unfortunately in some cases, the infant dies despite early therapy.

\section{Conclusions}

Because of the serious adverse neurological outcomes of GBS meningitis, there is the need for prevention of GBS infections and for ongoing developmental follow-up for GBS meningitis survivors.

\section{Conflict of Interests}

The authors declare that there is no conflict of interests regarding the publication of this paper.



Figure 22. Coronal section: echogenic widening of posterior interhemispheric fissure.

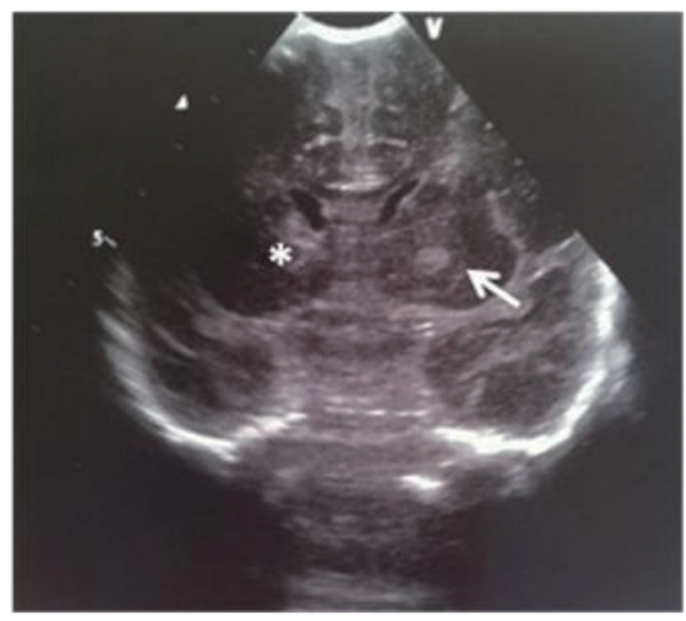

Figure 23. Coronal section: asymmetric lateral ventricles; rounded echodensity in the left thalamus (arrow) and incipient cavitation of both left caudate nucleus and thalamus (star).

\section{References}

1. Hernandez MI, Sandoval CC, Tapia JL, Mesa T, Escobar R, Huete I, Wei XC, et al. Stroke patterns in neonatal group B streptococcal meningitis. Pediatr Neurol. 2011;44(4):282-288.

2. Chung MY, Ko DJ, Chen CC, Huang CB, Chung CH, Chen FS, Hwang KP. Neonatal group B streptococcal infection: a 7-year experience. Chang Gung Med J. 2004;27(7):501-508

3. Le Doare K, Kampmann B. Breast milk and Group B streptococcal infection: vector of transmission or vehicle for protection? Vaccine. 2014;32(26):3128-3132.

4. Lin FY, Whiting A, Adderson E, Takahashi S, Dunn DM, Weiss R, Azimi PH, et al. Phylogenetic lineages of inva- 
sive and colonizing strains of serotype III group B Streptococci from neonates: a multicenter prospective study. J Clin Microbiol. 2006;44(4):1257-1261.

5. Trends in perinatal group B streptococcal disease - United States, 2000-2006. MMWR Morb Mortal Wkly Rep. 2009;58(5):109-112.

6. Doran KS, Benoit VM, Gertz RE, Beall B, Nizet V. Late-onset group B streptococcal infection in identical twins: insight to disease pathogenesis. J Perinatol. 2002;22(4):326-330.

7. van Sorge NM, Quach D, Gurney MA, Sullam PM, Nizet V, Doran KS. The group B streptococcal serine-rich repeat 1 glycoprotein mediates penetration of the bloodbrain barrier. J Infect Dis. 2009;199(10):1479-1487.

8. Seo HS, Minasov G, Seepersaud R, Doran KS, Dubrovska I, Shuvalova L, Anderson WF, et al. Characterization of fibrinogen binding by glycoproteins Srr1 and Srr2 of Streptococcus agalactiae. J Biol Chem. 2013;288(50):3598235996.

9. Tazi A, Disson O, Bellais S, Bouaboud A, Dmytruk N, Dramsi S, Mistou MY, et al. The surface protein HvgA mediates group B streptococcus hypervirulence and meningeal tropism in neonates. J Exp Med. 2010;207(11):23132322.

10. Magalhaes V, Andrade EB, Alves J, Ribeiro A, Kim KS, Lima M, Trieu-Cuot P, et al. Group B Streptococcus hijacks the host plasminogen system to promote brain en- dothelial cell invasion. PLoS One. 2013;8(5):e63244.

11. Govaert P, De Viries SL. An atlas of neonatal brain sonography. 2nd edition. Mac Keith Press. 2010.

12. Yikilmaz A, Taylor GA. Sonographic findings in bacterial meningitis in neonates and young infants. Pediatr Radiol. 2008;38(2):129-137.

13. Ansong AK, Smith PB, Benjamin DK, Clark RH, Li JS, Cotten CM, Mangum B, et al. Group B streptococcal meningitis: cerebrospinal fluid parameters in the era of intrapartum antibiotic prophylaxis. Early Hum Dev. 2009;85(10 Suppl):S5-7.

14. Baker CJ. The spectrum of perinatal group B streptococcal disease. Vaccine. 2013;31(Suppl 4):D3-6.

15. Centres for Disease Control and Prevention (CDC): Prevention of perinatal group B streptococcal disease. MMWR Morb Mortal Wkly Rep. 2002;51:1-6.

16. Natarajan G, Johnson YR, Zhang F, Chen KM, Worsham MJ. Real-time polymerase chain reaction for the rapid detection of group B streptococcal colonization in neonates. Pediatrics. 2006;118(1):14-22.

17. Ramjattan KC. Late-onset group B streptococcus disease in the late-preterm neonate. J Pediatr Health Care. 2014;28(4):361-365.

18. Libster R, Edwards KM, Levent F, Edwards MS, Rench MA, Castagnini LA, Cooper T, et al. Long-term outcomes of group B streptococcal meningitis. Pediatrics. 2012;130(1):e8-15. 